\title{
Comparison of Stress and Social Support between Veterans and Non-Veterans; Case Study of Urmia City, Iran
}

\section{ART ICLE INF O}

\section{Article Type}

Descriptive Study

\section{Authors}

Hasani Tabatabai L. ${ }^{1} M A$, Shaker Dioulagh A.* $P h D$

How to cite this article
Hasani Tabatabai L, Shaker Di-
oulagh A. Comparison of Stress
and Social Support between Ve-
terans and Non-Veterans; Case
Study of Urmia City, Iran. Iranian
Journal of War \& Public Health.
2017;9(3):141-146.

*Psychology Department, Humanities Faculty, Urmia Branch, Islamic Azad University, Urmia, Iran ${ }^{1}$ Psychology Department, Humanities Faculty, Urmia Branch, Islamic Azad University, Urmia, Iran

\section{Correspondence}

Address: Psychology Department, 3rd Floor, Faculty of Humanities, Urmia Branch, Islamic Azad University, Basij Boulevard, Urmia, Iran Phone: +98 (44) 33688119 Fax: +98 (44) 33688119 ali.shaker2000@gmail.com

\section{Article History}

Received: January 29, 2017

Accepted: April 18, 2017

ePublished: July 27, 2017

\section{A B S T R A C T}

Aims Social stress and social support are factors that affect people's mental health. The purpose of this study was to compare social support and social stress among spinal cord and chemical veterans and ordinary people.

Instrument \& Methods This causal-comparative research was conducted in 2016 among the spinal cord and chemical veterans who received SCI or chemical injuries through the Medical Commission of the Martyr Foundation and the Veterans Affairs of the emperors and ordinary people in Urmia City, Iran. 130 samples (30 spinal cord injured, 50 chemical veterans and 50 normal people) were studied. The sampling method was census for spinal cord injured, simple randomized for chemical veterans and clustered for normal people. The research tool was the Duke Social Protection Scale and Social Stress. Data analysis was done by SPSS 22 software using multivariate analysis of variance and Scheffe post hoc tests.

Findings There was a significant difference in social support between chemical veterans and normal people and also between chemical veterans and spinal cord injured $(\mathrm{p}<0.05)$, but there was no significant difference between the normal group and spinal cord injured ( $p>0.05)$. In addition, there was a significant difference in social stress between chemical veterans and normal people $(\mathrm{p}<0.05)$, but spinal cord injured had no significant differences with normal people and chemical veterans ( $\mathrm{p}>0.05)$.

Conclusion Social support and social stress are different among spinal cord injured, chemical veterans and ordinary people.

Keywords Social Support; Social Stress; Spinal Cord Injuries; Veterans

\section{I T A T I O N L I N KS}

[1] Comparison the effect of training of conflict resolution and relaxation on quality of life's spouses of war veterans affected Post traumatic stress disorder with major ... [2] Qualification of studies conducted on the mental health status ofveterans:Asystematic... [3] Depression and suicidal tendencies in veterans with spinal injury hospitalized in Tehran and nursing home patients and ... [4] Health care service satisfaction among chemical warfare survivors with severe ophthalmic ... [5] Depression after spinal cord injury: Is it ... [6] Relation between perceived social support and health promotion behaviors in chemical veterans in Ilam ... [7] Association o depressive symptoms and social support on blood pressure among urban African American ... [8] HPostpartum Taiwanese women: their postpartum depression, social support and healthpromoting lifestyle ... [9] The relationship between social support ... [10] Distinct relationship between hardiness, perceived stress and social support and ... [11] Social support andlife satisfaction in spinal cord injury to one year after during and up inpatient ... [12] Factors influencing the link between social anxiety and peer acceptance: Contributions of social skills and close friendships during ... [13] Group social skills training or cognitive group therapy as the clinical treatment of choice for generalized ... [14] Attentional bias away from positive social information ... [15] Relationship between perfectionism, perceived ... [16] Anxiety, depression, and posttraumatic stress in Iranian ... [17] Stress coping strategies and social ... [18] Relationship between social anxiety, hope ... [19] The relationship between social support and occupational ... [20] Investigating the Association between social ... [21] Comparing social support and social anxiety between ... [22] Comparison of depression, anxiety and stress ... [23] Comparing the perceived social support and religiosity in ... [24] Assessment of relationship quality of life ... [25] Relation between social support and coping with ... [26] Evaluation of ptsd in sardasht survivors ... 
جنبههاى مختلف زندگى فرد و خانواده وى را تحت تاثير قرار

دهد]"].

تحقيقات نشان داده است كه جانبازان شيميايى نسبت به ساير

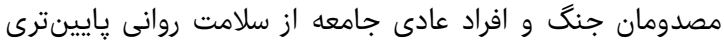

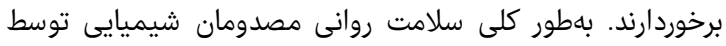

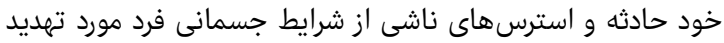

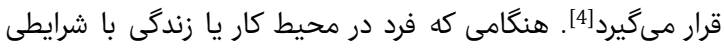

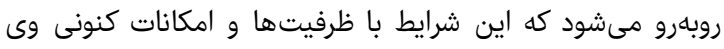

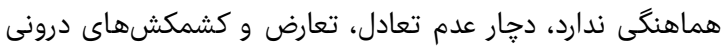

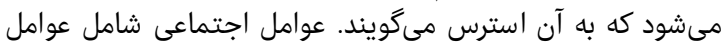

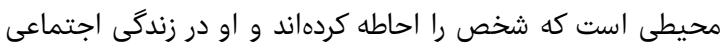

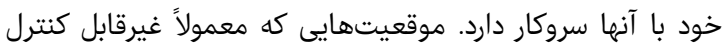

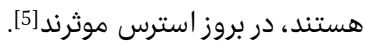

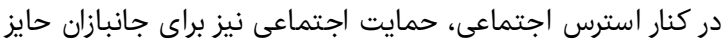

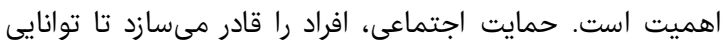

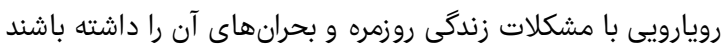

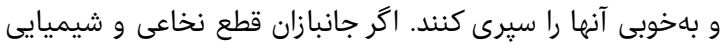

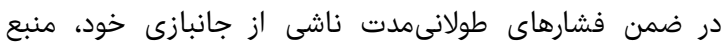

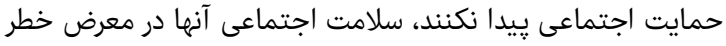

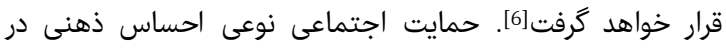

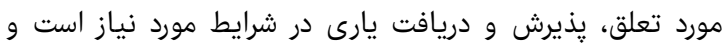

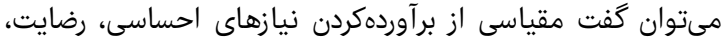

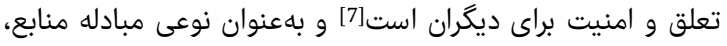

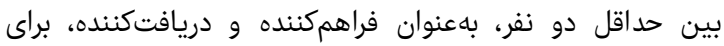

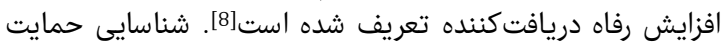

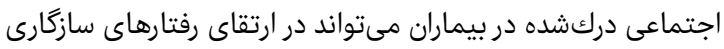

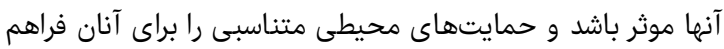

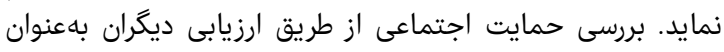

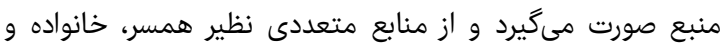

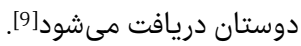

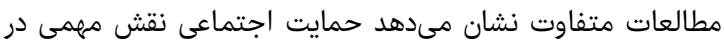

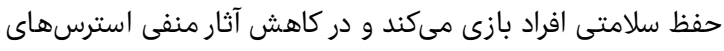

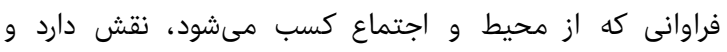

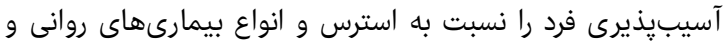

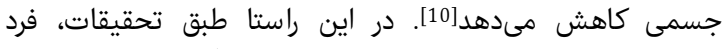

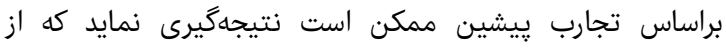

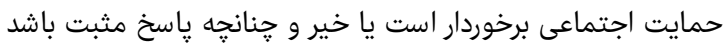

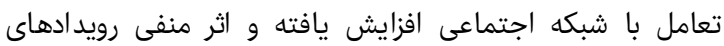

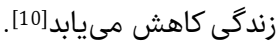

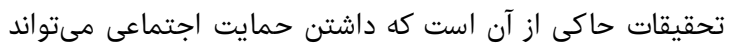

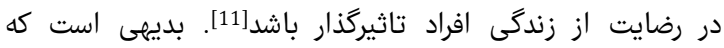

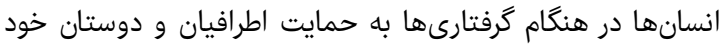

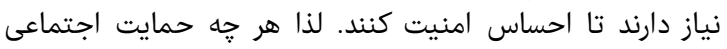

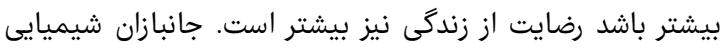

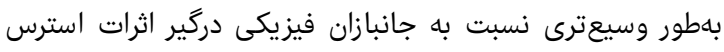

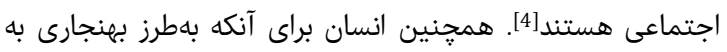

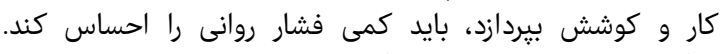

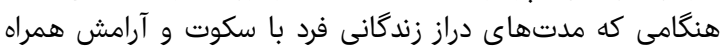

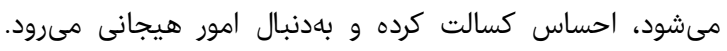

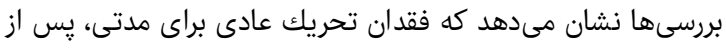

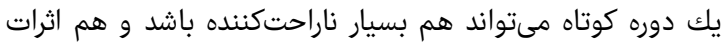

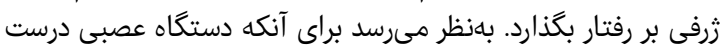

دوره 9، شماره ץ، تابستان عوسا
مقايسه حمايت و استرس اجتمانماعى بين بين جانبازان و غيرجانبازان؛ مطالعه موردى شهر اروميت إيتريه

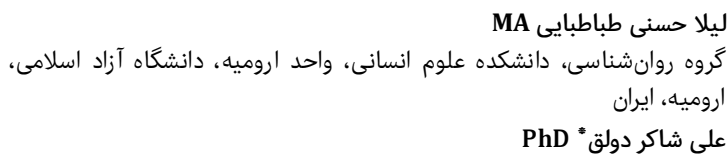

كروه روانشناسى، دانشكده علوم انسانى، واحد اروميه، دانشكاه آزاد اسلامى،

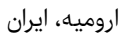

جكيده

اهّداف: استرس اجتماعى و حمايت اجتماعى از عوامل تاثيرگذار بر

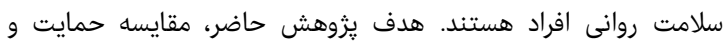

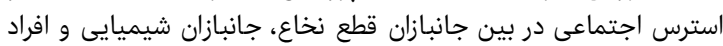

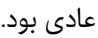

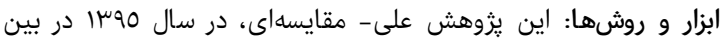

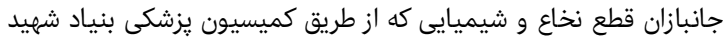

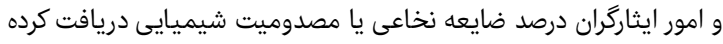

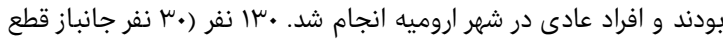

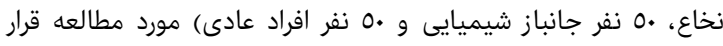

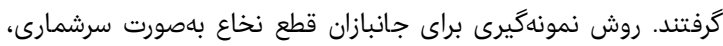

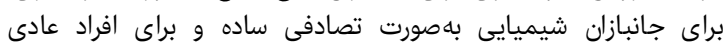

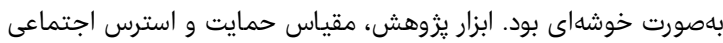

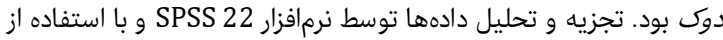

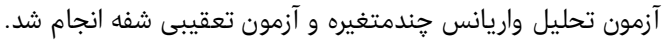

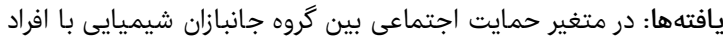

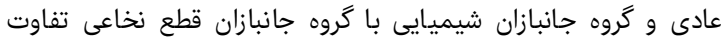

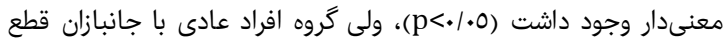

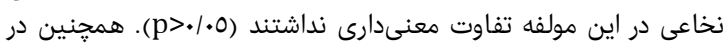

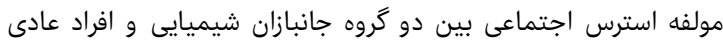

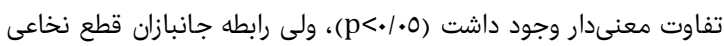

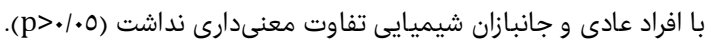

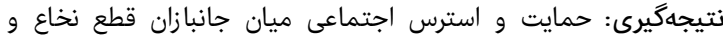

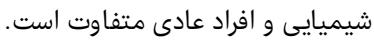

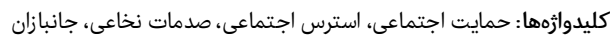

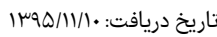

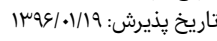

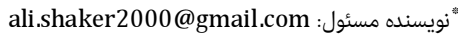

مقدمه

جنگ يكى از عوامل تاثيرگذار بر ميزان شيوع، زمان شروع و سير

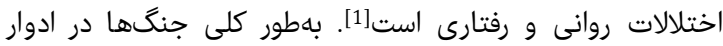

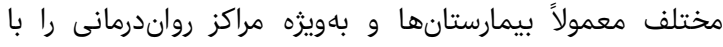

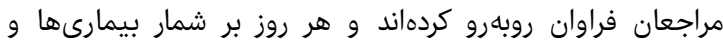

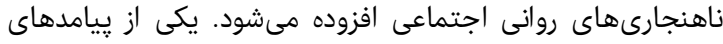

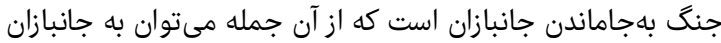

قطع نخاعى و جانبازان شيميايى اشاره كرد [2].

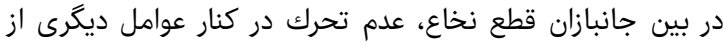

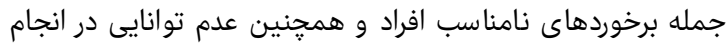

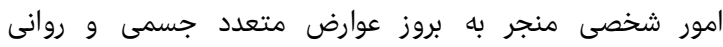

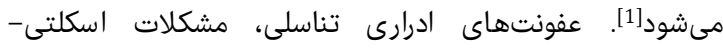

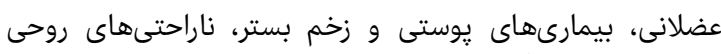

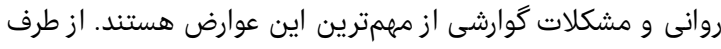

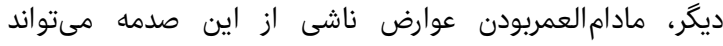




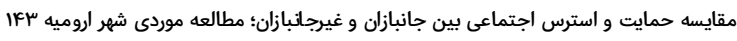

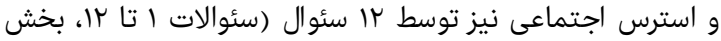
دو) مورد ارزيابى قرار مى إنيرد.

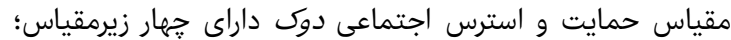

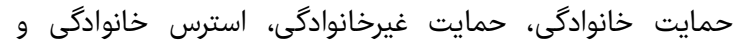

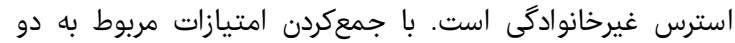

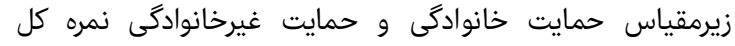

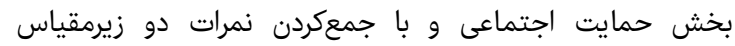

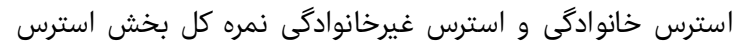

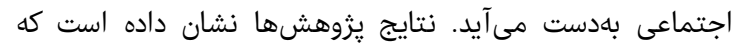

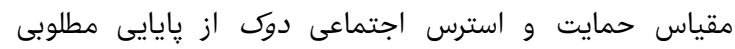

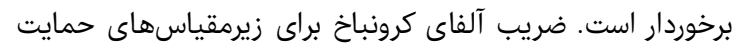

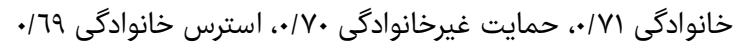

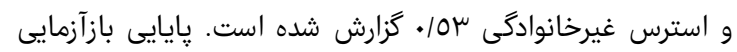

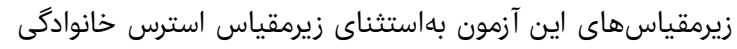

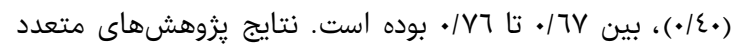

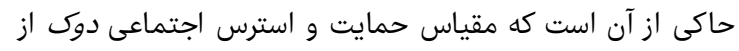

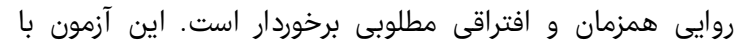

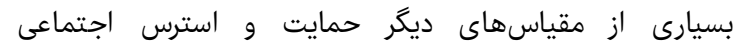

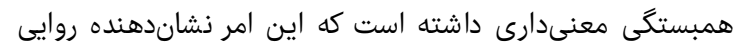

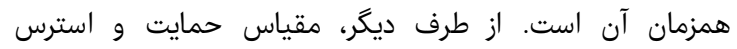

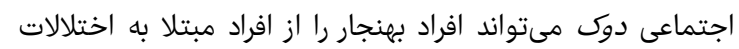

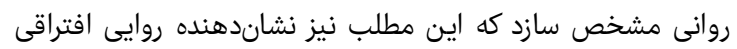

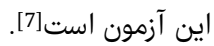

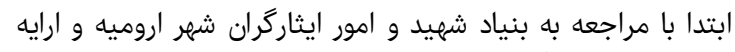

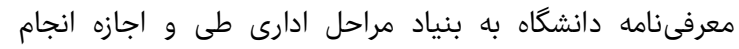

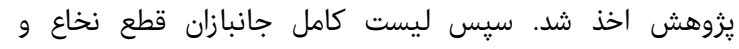

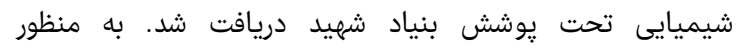

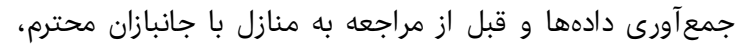

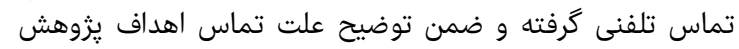

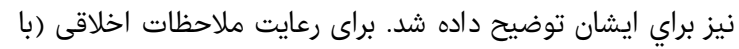

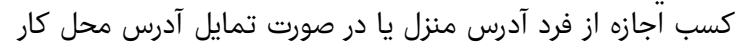

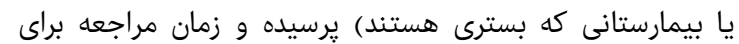

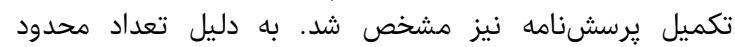

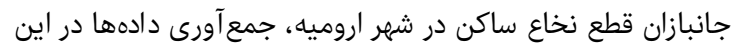

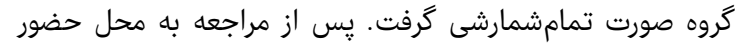

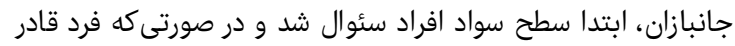

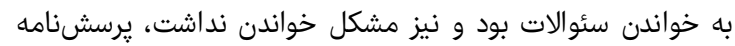

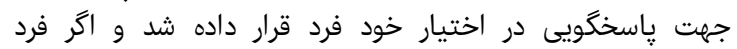

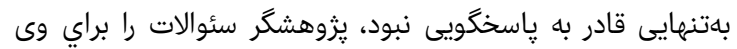

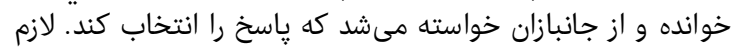

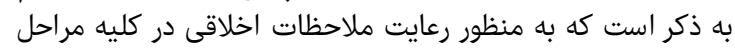

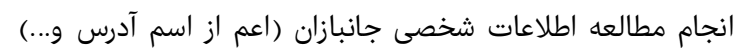

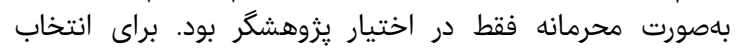

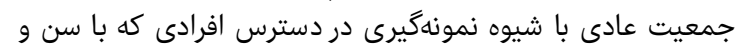

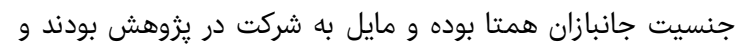

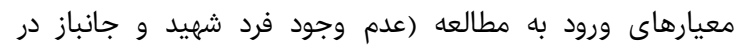

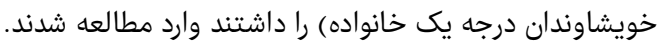

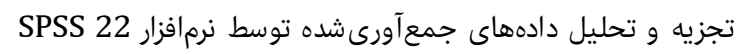

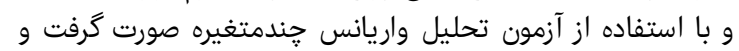

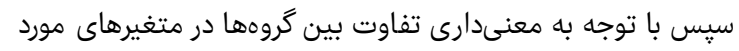

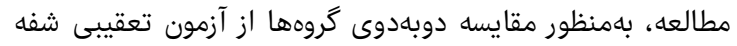

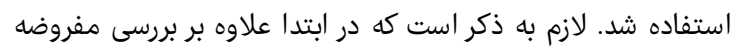

كار كند بايستى ميزان معينى تحريك به آن وارد شود، اما در عين

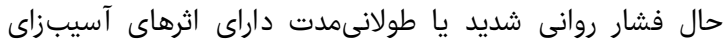

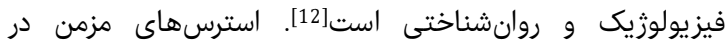

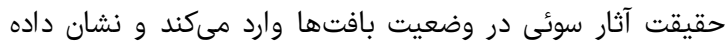

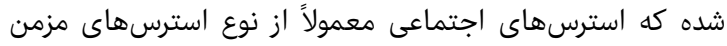

هستند[13].

استرس اجتماعى به استرس يا هراس كه در موقعيتهاى

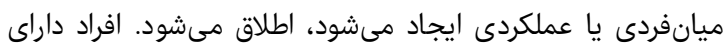

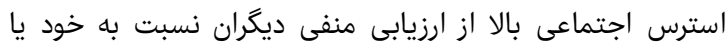

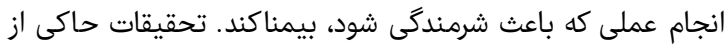

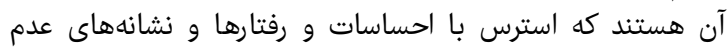

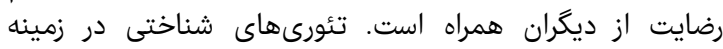

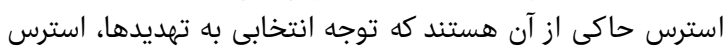

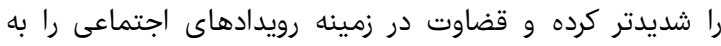
انحراف مىكشاند[14].

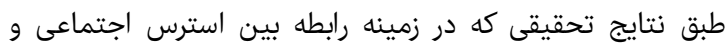

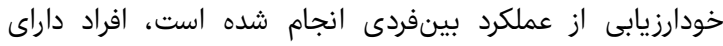

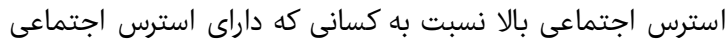

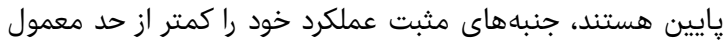

ارزيابى مىكنند [15].

با توجه به بررسىهاى انجامشده، متاسفانه تاكنون محققان به

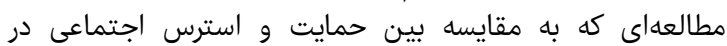

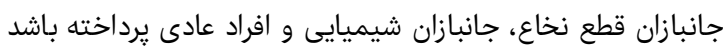

دست نيافتهاند.

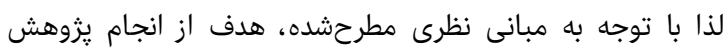

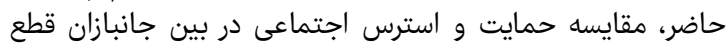
نخاع، جانبازان شيميايى و افراد عادى بود.

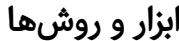

يثرهش حاضر از نوع على- مقايسهاى است كه از تير تا مهر

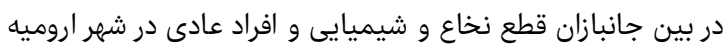

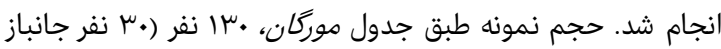

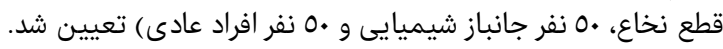

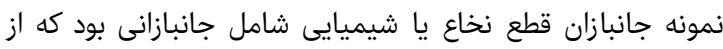

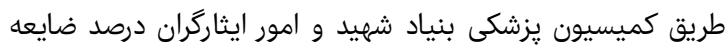

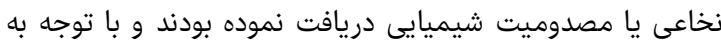

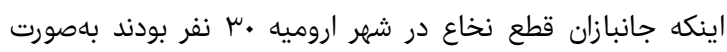

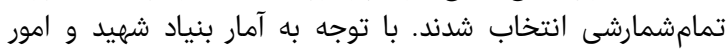

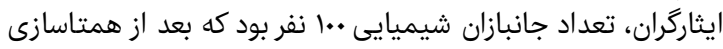

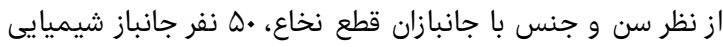

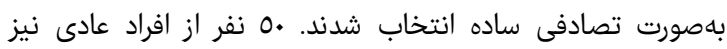

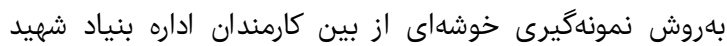

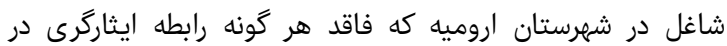

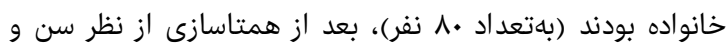

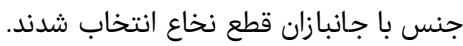

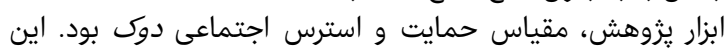

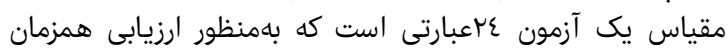

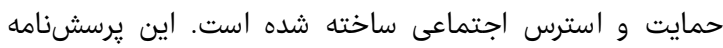

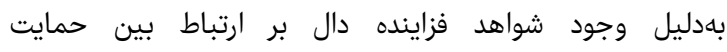

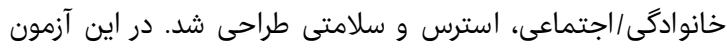

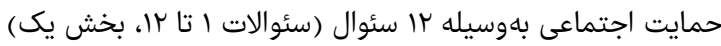


همجنين در متغير حمايت اجتماعى بين كروه جانبازان شيميايى

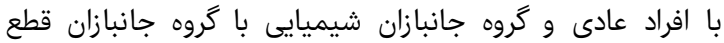

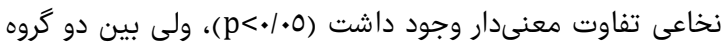

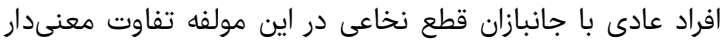

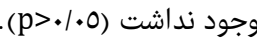

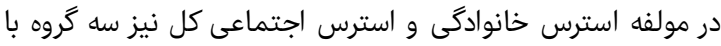

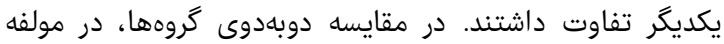

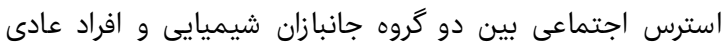

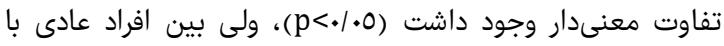

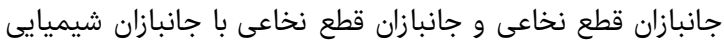

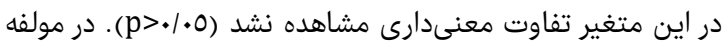

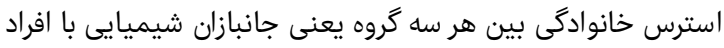

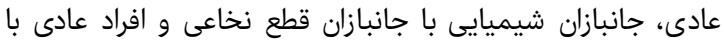

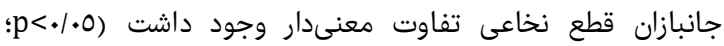

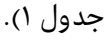

أifF

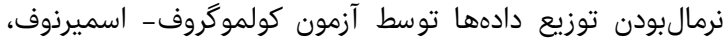

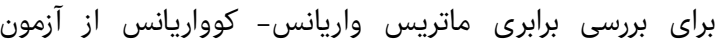
امباكس و براى بررسى همكنى متغيرها در سه كروه از آزمون لون آرن آرن

استفاده شد.

يافتهها

تمام افراد مورد مطالعه، مرد بودند و ميانكين سنى آنها .

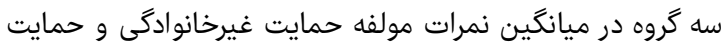

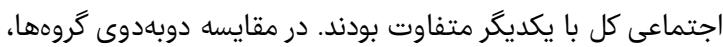

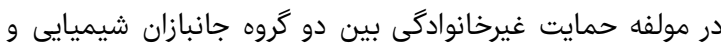

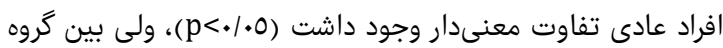

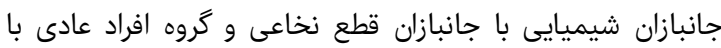

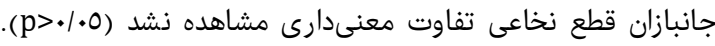

جدول () مقايسه ميانكين آمارى نمرات حمايت و استرس اجتماعى و زيرمقياس هاى آنها در سه گروه با استفاده از آزمون تحليل واريانس جندمتغيره

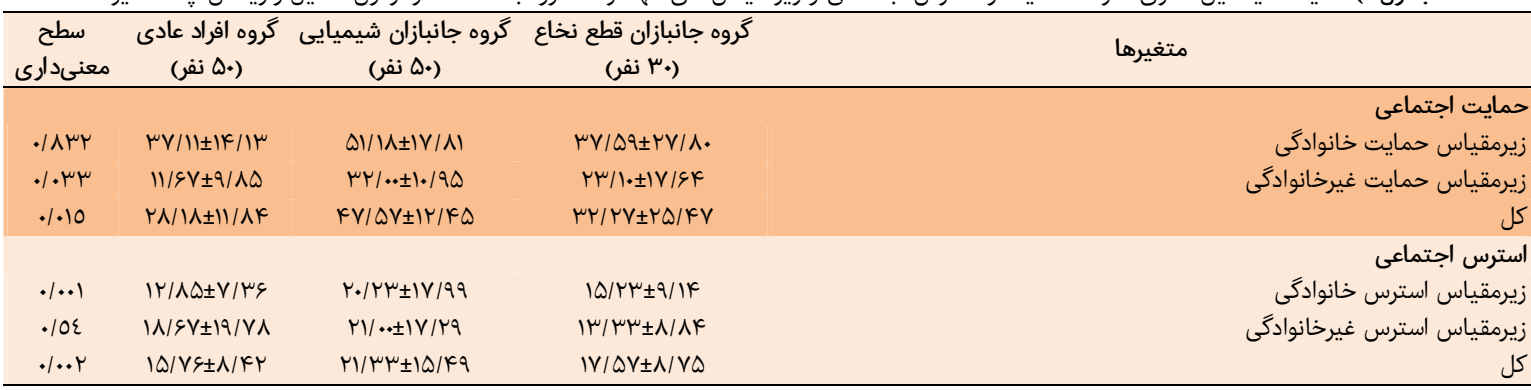

داد[10]. يزوهشها نشان دادهاند افرادى كه داراى حمايت اجتماعى دئ

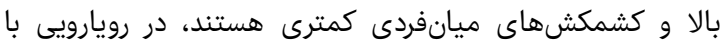
رخدادهاى فشارزاى زندگى بيشتر ايستادگى مى مئى

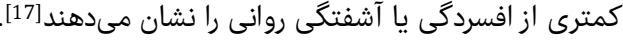

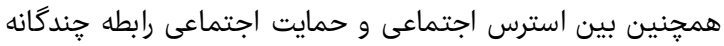

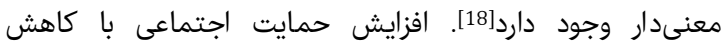

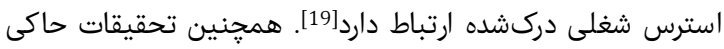

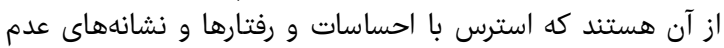

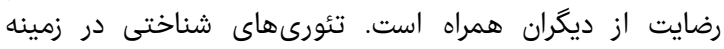

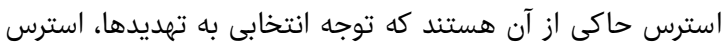

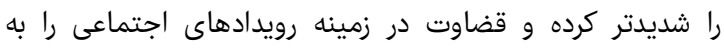

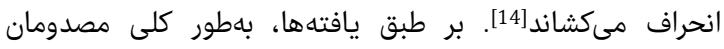

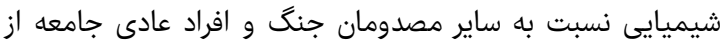

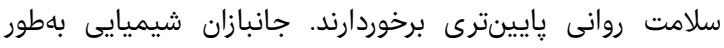

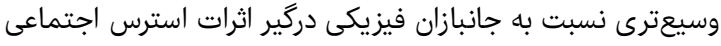

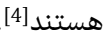

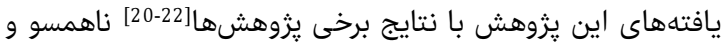

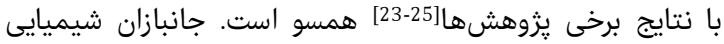

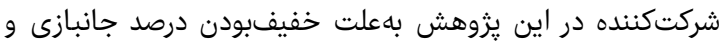

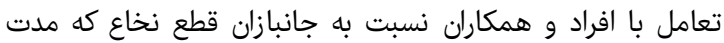

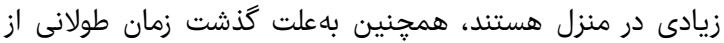

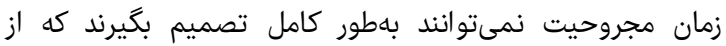

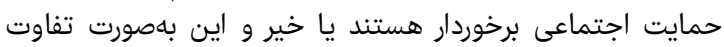

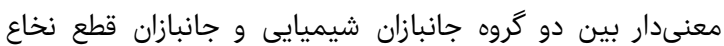

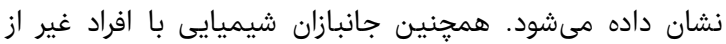

براساس نتايج حاصل، بين دو گروه جانبازان شيميايى و افراد عادى

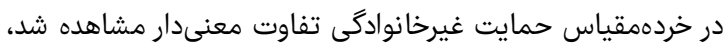

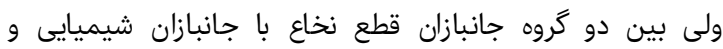

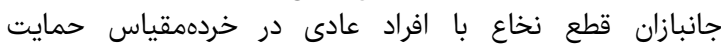

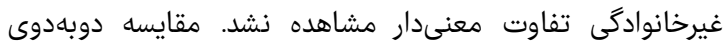

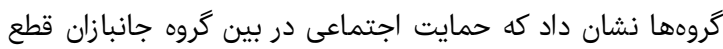

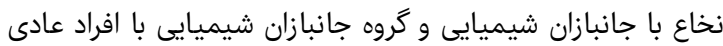

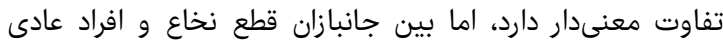
تفاوت معنىدار وجود ندارد.

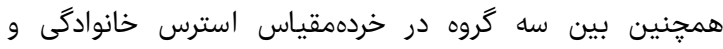

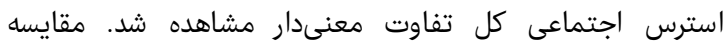

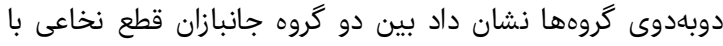

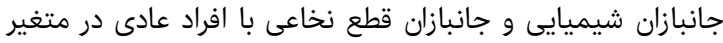

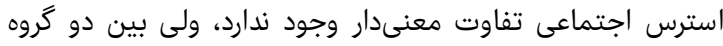

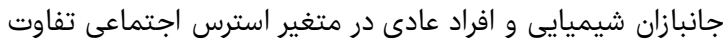

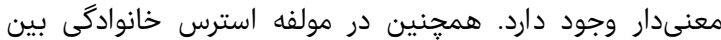

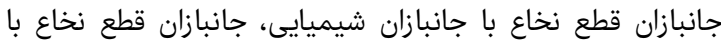

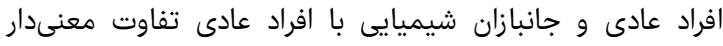

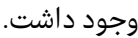
در تبيين اين يافتهها مى داتوان كفت حمايت اجتماعى در كاهش إشاء

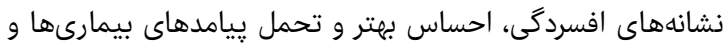

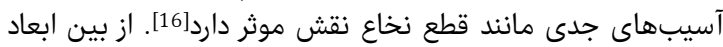

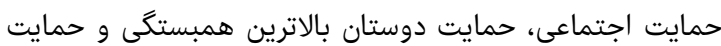

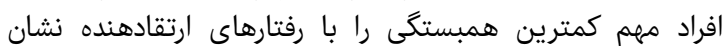


1- Parande A, Siratinir M, Khaghanizadeh M, Karimizarchi AA. Comparison the effect of training of conflict resolution and relaxation on quality of life's spouses of war veterans affected Post traumatic stress disorder with major depression disorder. J Fundam Ment Health. 2007;9(33-34):67-76. [Persian]

2- Ahmadi Kh, Shahi R, Habibi M. Qualification of studies conducted on the mental health status of veterans: A systematic review. J Behav Sci. 2011;5(3):217-24. [Persian]

3- Matinisadr MR. Depression and suicidal tendencies in veterans with spinal injury hospitalized in Tehran and nursing home patients and healthy subjects [Dissertation]. Tehran: Tehran Psychiatric Institute; 1991. [Persian]

4- Mousavi B, Soroush M, GanjParvar Z. Health care service satisfaction among chemical warfare survivors with severe ophthalmic complications. Iran J War Public Health. 2008;1(1):12-21. [Persian]

5- Frank G, Elliot TR, Corcoran JR, Wonderlich SA. Depression after spinal cord injury: Is it necessary?. Clin Psych J. 1987;7(6):611-630.

6- Chenary R, Noroozi A, Noroozi R. Relation between perceived social support and health promotion behaviors in chemical veterans in Ilam province on 2012-13. Iran J War Public Health. 2013;6(1):1-10. [Persian]

7- Wu ChY, Prosser R, Taylor J. Association o depressive symptoms and social support on blood pressure among urban African American women and girls. J Am Acad Nurs Pract. 2010;2(2):694-704.

8- Chen CM, Kuo SF, Chou YH, Chen HC. Postpartum Taiwanese women: their postpartum depression, social support and healthpromoting lifestyle profiles. J Clin Nurs. 2007;16(8):1550-60.

9- Riyahi ME, Ali Verdi Nia A, Pourhossein SZ. The relationship between social support and mental health. Soc Welf. 2008;10(39):85-121. [Persian]

10- Johari J, Asgari S, Yazdanbakhsh K. Distinct relationship between hardiness, perceived stress and social support and marital adjustment [Dissertation]. Tehran: Razi University; 2013. [Persian]

11- Van leeuwen MC, Post WM, Van Asbeck WF, Vander Woude HV, de Groot S, Lindeman E. Social support andlife satisfaction in spinal cord injury to one year after during and up inpatient rehabilitation. J Psychol. 2010;42(3):265-71.

12- Greco LA. Factors influencing the link between social anxiety and peer acceptance: Contributions of social skills and close friendships during middle childhood. Behav Ther. 2005;36(2):197-205.

13- van Dam-Baggen R1, Kraaimaat F. Group social skills training or cognitive group therapy as the clinical treatment of choice for generalized social phobia? J Anxiety Disord. 2000;14(5):437-51.

14- Taylor CT, Bomyea J, Amir N. Attentional bias away from positive social information mediates the link between social anxiety and anxiety vulnerability to a social stressor. J Anxiety Disord. 2010;24(4):403-8.

15- Behroozi N, Shahani Yeylaq M, Pourseyed SM. Relationship between perfectionism, perceived stress and social support with academic burnout. J Manag Sys. 2012;20(4):83-102. [Persian]

16- Hashemian F, Khoshnood K, Desai M, Falahati F, KaslS, Southwick S. Anxiety, depression, and
خانواده بيشتر از جانبازان قطع نخاع تماس دارند و حمايت

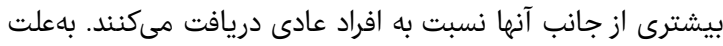

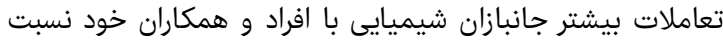

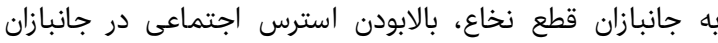

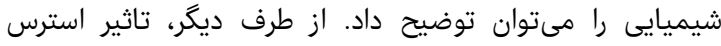

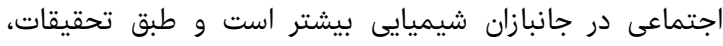

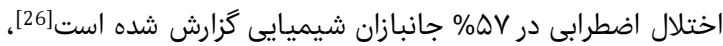
در صورتى كه جانبازان قطع نخاع تماس كمترى جانى با افراد ديكر و

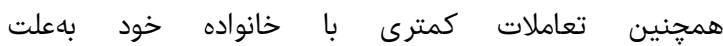
محدوديتهايشان دارند.

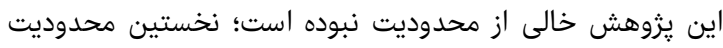

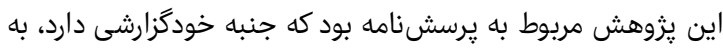

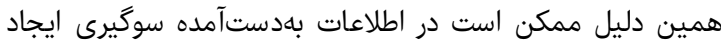

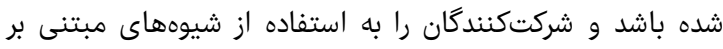

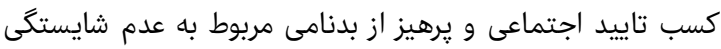

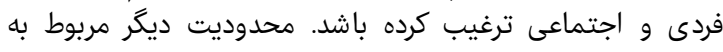

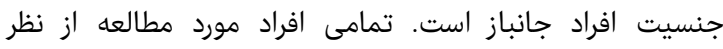
جنسيت مرد بودند، در نتيجه قادر به بررسى اثر جنسيت أثراديت در متغيرها

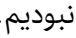

براساس اين محدوديتها يِيشنهاد مىشود بهجاي استفاده از ابزار

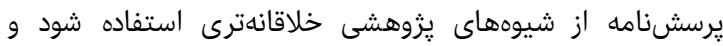

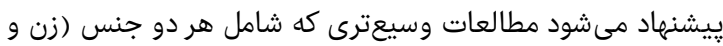
مرد) باشد، انجام بكيرد.

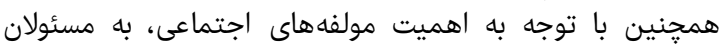

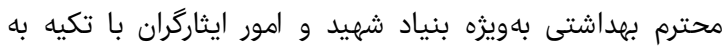

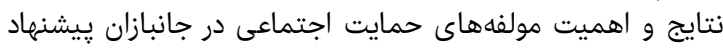

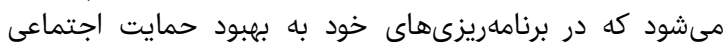

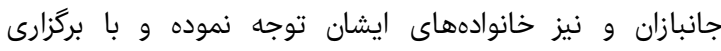

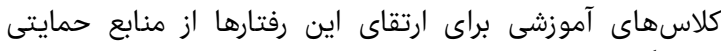

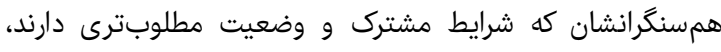

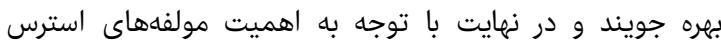

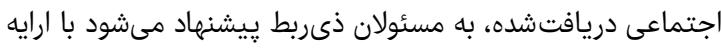

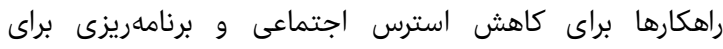
دسترسى راحتتر جانبازان به مشاوره در رفع اين مورد سعى نمايند.

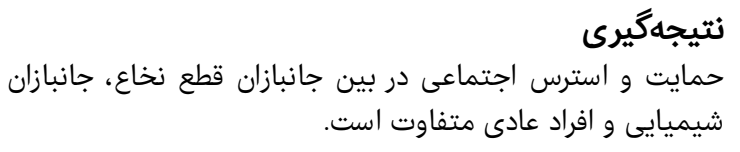

تشكر و قدردانى: از تمامى كاركنان بنياد شهيد و امور ايثاركران

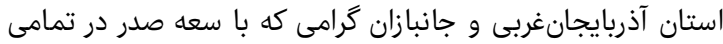

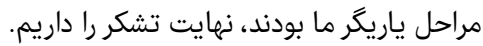

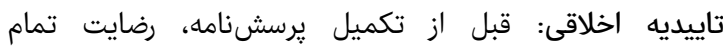

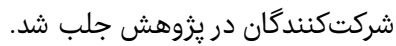

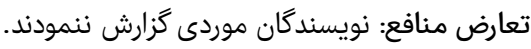

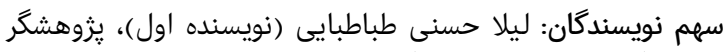

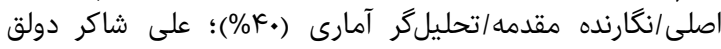

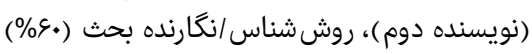

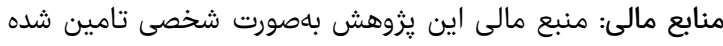


2015;16(2):43-52. [Persian]

22- Ahmadi Kh, Reshadatjo M, Karami Gh. Comparison of depression, anxiety and stress rate between chemical warfare victims and healthy persons in Sardasht Iran. J Babol Univ Med Sci. 2010;12(1):44-50. [Persian]

23- Bayrami M, Andalib Koraim M, Poureamaeli A, Mohamadibakhsh L. Comparing the perceived social support and religiosity in people with post-traumatic disorders, their spouses control group. J Kermanshah Univ Med Sci. 2013;17(1):68-75. [Persian]

24- Safavi M, Mahmoudi M, Akbarnatajbishe K. Assessment of relationship quality of life and coping skills in spouses of chemical devotees with pulmonary complications due to sulfur mustard in Tehran in 2006. Daneshvar. 2010;17(87):9-18. [Persian]

25- Akouchekian Sh, Roohafza HR, Hasan zadeh A, Mohammad Sharifi H. Relation between social support and coping with stress in nurses in psychiatric ward. J Guilan Univ Med Sci. 2009;18(69):41-6. [Persian]

26- Ahmadi Kh, Reshadatjoo M, Karami GR. Evaluation of ptsd in sardasht survivors of chemical warfare. J Urmia Univ Med Sci. 2010;21(1):1-9. [Persian]

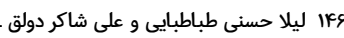
posttraumatic stress in Iranian survivors of chemical warfare. JAMA. 2006;296(5):560-6.

17- Ebrahimi A, Bolhari J, Zolfaghari F. Stress coping strategies and social support in depressive veterans with spinal cord injury. Iran J Psychiatry Clin Psychol. 2002;8(2):40-8. [Persian]

18- Asgare P, Sharafedini H. Relationship between social anxiety, hope and social support with subjective wellbeing in postgraduate students of Khouzestan Science and Research Unit. J Soc Psycol. 2008;3(9):25-36. [Persian]

19- Graf FA. The relationship between social support and occupational stress among police officers. J Police Sci Adm. 1986;14(3):178-86.

20- Salah Adin S. Investigating the Association between social support and social health among psychiatric veterans' wives in Tehran. Q J Soc Work. 2014;3(1):3443. [Persian]

21- Jadidi M, Safari S, Faramarzi S, Jadidi M, Jamali S. comparing social support and social anxiety between mothers of children with special needs and mothers of normal children. Knowl Res Appl Psychol. 